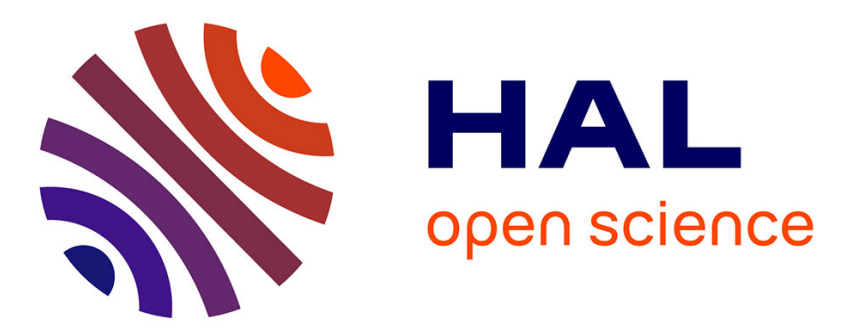

\title{
Recording performances in perpendicular magnetic patterned media
}

M Asbahi, J Moritz, B Dieny, C Gourgon, C Perret, R.J.M. van de Veerdonk

\section{To cite this version:}

M Asbahi, J Moritz, B Dieny, C Gourgon, C Perret, et al.. Recording performances in perpendicular magnetic patterned media. Journal of Physics D: Applied Physics, 2010, 43 (38), pp.385003. 10.1088/0022-3727/43/38/385003 . hal-00597831

\section{HAL Id: hal-00597831 https://hal.science/hal-00597831}

Submitted on 2 Jun 2011

HAL is a multi-disciplinary open access archive for the deposit and dissemination of scientific research documents, whether they are published or not. The documents may come from teaching and research institutions in France or abroad, or from public or private research centers.
L'archive ouverte pluridisciplinaire HAL, est destinée au dépôt et à la diffusion de documents scientifiques de niveau recherche, publiés ou non, émanant des établissements d'enseignement et de recherche français ou étrangers, des laboratoires publics ou privés. 


\title{
Recording Performances in Perpendicular Magnetic Patterned Media
}

\author{
M. Asbahi, J. Moritz and B. Dieny ${ }^{(a)}$ \\ C. Gourgon and C. Perret ${ }^{(b)}$ \\ R.J.M. van de Veerdonk ${ }^{(\mathrm{c})}$
}

(a) SPINTEC, UMR8191 CEA/CNRS/UJF/Grenoble-INP ; CEA/INAC, 17 rue des Martyrs, 38054 Grenoble Cedex, France.

(b) Laboratoire des Technologies de la Microélectronique, 17 Av. des Martyrs (c/o CEA Grenoble) F-38054 Grenoble, France

(c) Seagate Recording Media Operations, 47010 Kato Road, Fremont, CA 94538,USA.

\begin{abstract}
:
We report on the recording performances and signal-to-noise ratio analyses of perpendicular magnetic bit-patterned media. Two different types of magnetic samples are investigated. They differ by the way that they were patterned (nano-imprint versus e-beam lithography) as well as their magnetic properties $(\mathrm{Co} / \mathrm{Pt}$ multilayers and $\mathrm{CoCrPt}$ alloy are the recording layers). By using a contact read/write quasi-static tester, we were able to characterize the write windows, the bit error rates, and measure the signal-to-noise ratio. The influence of magnetic properties and media microstructure on the writing processes is studied. We show also that the lithographical method used to replicate the media induces more or less noise due to structural distributions.
\end{abstract}

PACS: 75.50.Ss; 85.70.Li; 75.30.Gw; 75.70.Ak

Corresponding author: jerome.moritz@cea.fr 


\section{Introduction}

To extend the magnetic storage density beyond the superparamagnetic limit of conventional media, various approaches were proposed including energy assisted magnetic recording (HAMR or MAMR) and bit patterned media (BPM) [1]. The main issue with BPM is to find ways to produce them with a high throughput, high structural quality and at a low cost [2]. Therefore, considerable research efforts have been devoted to the fabrication of patterned media [3]. The present study aims to better understand the recording performances and noise properties of these media [4]-[6], in particular on the influences of both material properties and patterning method on the writing synchronization and noises.

In contrast to continuous media, bits position in patterned media is predefined by patterning process. Therefore to ensure a minimum bit error rate (BER), the write pulse has to be synchronized to the bits themselves. If the write current is switched before or after the head has passed above the targeted dot, the bit is not addressed correctly. However during a time margin, called the write window (WW), writing is $100 \%$ efficient for an optimal write current. If the head field is switched outside the WW, a written-in error occurs. The write synchronization can be achieved by an internal clock, which is used to control the write current and follow the bits position [7]. But the manufacturing process of patterned media induces distributions in sizes and in spacing of the dots, so the internal clock may only accomplish an average synchronization. Albrecht et al. have measured a WW of S, where S is the dots spacing, in focused ion beam patterned structures [8]. So a distribution of $\mathrm{S}$ can reduce considerably the WW width. It has also been demonstrated by Livshitz et al. the WW width dependence on material parameters [9]. The authors have clearly shown the WW width was reduced, when a distribution of anisotropy field is considered. Therefore both material parameters and patterning process have to be well controlled to avoid written-in errors. 
Considering then that all bits are correctly written, imperfections of the media, as distributions in sizes and in spacing of the bits, deteriorate the system read-back performances [10][11]. These distributions result in a signal-to-noise ratio (SNR) expressed as [12]:

$$
S N R=\frac{1}{\left(\pi^{2}-7.5\right)\left(\frac{\sigma_{S}}{S}\right)^{2}+\left(R \frac{\sigma_{D}}{D}\right)^{2}}
$$

Where $\frac{\sigma_{S}}{S}$ and $\frac{\sigma_{D}}{D}$ are the dots spacing and the dots size fluctuations respectively, and $\mathrm{R}$ a numerical factor $1<\mathrm{R}<2$ depending on the head geometry. To reach the Tbit/in ${ }^{2}$ density, the dots size and spacing have to be for instance 15 and $10 \mathrm{~nm}$ respectively. A 20db SNR requires $\frac{\sigma_{S}}{S}$ and $\frac{\sigma_{D}}{D}<0.05-0.1$, which implies high quality control of the patterning processes.

In this paper, we report on an experimental study focused on the recording and noise characteristics of two types of patterned media. We show that both material and manufacturing processes influence the BER and the SNR.

\section{General properties of the patterned media}

We have investigated two different samples: sample A consists of $1 \mathrm{~mm}^{2}$ pre-patterned square array of $160 \times 80 \mathrm{~nm}^{2} \mathrm{Si}$ dots, fabricated by thermal nano-imprint lithography and reactive ion etching, covered in a second step by perpendicular-to-plane magnetized $\mathrm{Pt}_{15 \mathrm{~nm}} /\left(\mathrm{Co}_{0.6 \mathrm{~nm}} / \mathrm{Pt}_{1.8 \mathrm{~nm}}\right)_{4}$ multilayers; sample $\mathrm{B}$ consists of $100 \mathrm{~nm}$ diameter dots, spaced by $200 \mathrm{~nm}$, arranged in an hexagonal array written in circular tracks and made by e-beam and etching in a $20 \mathrm{~nm}$ thin perpendicular-to-plane magnetized CoCrPt alloy. Further details concerning the fabrication of sample A as well as its magnetic properties will be published elsewhere [13]. Figure 1a shows a Magnetic Force Microscopy (MFM) image of sample B in AC-demagnetized state. Each dot exhibits a mono-polar contrast associated with a 
perpendicular-to-plane magnetization; moreover the features remain single domain at zero field, the same being true in sample A [14].

In figure $1 \mathrm{~b}$ the decreasing field branches of the hysteresis loops of both samples measured by magneto-optical polar Kerr effect are depicted. The average coercive fields and the switching field distributions (SFD) are given in table 1. For sample A the coercive field of the dots varies between 0.173 and $0.389 \mathrm{~T}$, whereas sample B is more coercive, with an average switching field of about $0.694 \mathrm{~T}$.

Anisotropy of both samples was evaluated by measuring the in-plane saturation field as explained in reference 15. Sample B being an optimized CoCrPt alloy, its effective anisotropy is of about $9 \times 10^{5} \mathrm{~J} / \mathrm{m} 3$, almost one order of magnitude higher than sample A (see Table 1). Furthermore we have shown by atomic force microscopy that magnetic grains diameters are about $20 \mathrm{~nm}$ in sample A, $8 \mathrm{~nm}$ in sample B [16]. The reversal mode of the magnetization differs in both samples: for sample A (lower coercivity), nucleation and propagation of domain walls are the dominating processes [17] [18]; for sample B (higher coercivity), coherent reversal of the magnetization inside the grains is responsible for the high coercive field [19][20]. Note that the addition of $\mathrm{Cr}$ in $\mathrm{CoPtCr}$ alloy contributes to weakly coupled grains and favors coherent reversal for small grains.

The barrier height $\Delta \mathrm{E}$, which prevents the magnetization reversal, is thermally activated and depends on the reversal mode [21][22]. By magnetic after-effect measurements, we have already shown that $\Delta \mathrm{E}$ for sample $\mathrm{B}$ was larger than seen in sample $\mathrm{A}$ [13] (see table 1). Although the numerical values of both $\Delta \mathrm{E}$ are quite different, they largely fulfill the stability criterion of magnetic recording $\left(\Delta \mathrm{E}>40 \mathrm{k}_{\mathrm{B}} \mathrm{T}\right)$.

\section{Written-in errors and write windows}

To write bits sequences in both samples, we have employed a quasi-static contact tester, in 
which a commercial longitudinal GMR recording head is used to map the stray field. The head is fixed on a piezo stage allowing a scan window of $120 \times 120 \mu \mathrm{m}^{2}$ with a step resolution of $2 \mathrm{~nm}$ and a scan velocity of $5 \mu \mathrm{m} / \mathrm{s}$. The spatial resolution in reading mode is limited by the dimensions of the GMR recording head used (track-width of about 150nm). The head is placed in close contact with the sample, and by positioning the write pole at the vertical of the bits, it is possible to switch the magnetization of each dot individually. Then, the read-back signal can be obtained from the magneto-resistive sensor. The write bubble window is about $80 \times 150 \mathrm{~nm}^{2}$, which is close to the sizes of both studied arrays.

DC erased experiments have allowed the determination of the optimal write current before precisely testing our media in terms of signal-to-noise ratio and error rates. In each sample, a distribution of write current exists, which reflects the SFD mentioned above: the write current was experimentally measured within the range from 8 to $10 \mathrm{~mA}$ for sample A and from 15 to $17 \mathrm{~mA}$ for sample B.

Bits sequences were written in the media by positioning the write pole at the center of a track and by adjusting the piezo stage step to the desired bit periodicity. For instance, figure $2 \mathrm{a}, \mathrm{b}$ show sequences of alternating up and down bits on sample B. In figure $2 \mathrm{a}, 2 \mathrm{D}$ map of the GMR signal is depicted, whereas in figure $2 \mathrm{~b}$ the GMR signal along a track is plotted. To obtain such a perfect bit configuration, the write current, as well as the synchronization of the write pulse with the bit periodicity, were optimized. A phase shift between the array and the write pole, or, a too low or too high current, can induce written-in-errors. An artificial phase shift can result in a mis-synchronization, leading to an increase in the BER. The write window can be experimentally deduced from such a measurement. After reading a large part of a track with the GMR sensor, the write pole is precisely positioned at the center of a track and a phase shift is added in the down-track direction. The bit sequence used previously is written in the media and the result for sample A is shown in figure 3. The BER strongly depends on 
the phase shift, which is low for tracks 1,3, 5, 7 and large for tracks 2, 4, 6 (figure 3). The same writing process has been carried out for sample B with similar results.

In figure 4 the ratio of synchronized dots $v s$. the phase shift is plotted for both samples. The WW width can be measured in the previous graphics, where the BER $<5 \%$ : $66 \mathrm{~nm}$ for sample A and 170nm for sample B. Although both WW widths are close to the dots spacing $(80 \mathrm{~nm}$ and $200 \mathrm{~nm}$ for sample A and B respectively), it is difficult to synchronize $100 \%$ bits in sample A. Moreover, the transition length between $5 \%$ and $100 \%$ of BER is sharper for sample B than for sample A (50nm vs. $80 \mathrm{~nm}$ respectively). When the write pole is located above the trenches or at the dots edges, the bits reversal is still achievable (that is why the transition length is extended for a few tens of $\mathrm{nm}$ ). The reversal probability is clearly larger for the pre-patterned sample.

Boris Livshitz et al. showed that the WW width reduces when a distribution of easy axis in the media is considered and that the transition length between $5 \%$ and $100 \%$ of BER increases at the same time [9]. It is generally admitted in the literature a distribution of easy axis in the range $2.5-6^{\circ}$ for perpendicular-to-plane magnetized continuous media (see [23] and references herein). T. Thomson et al. explained that the SFD in patterned media arises from the distribution of intrinsic anisotropy rather than any fabrication related effects [24]. However we have previously shown by transmission electron microscopy that the Co/Pt multilayers deposited onto pre-patterned Si substrates exhibited a bending at the dots edges (see crosssection in figure 4 in reference 25). We have also demonstrated that the bending can reduce the local anisotropy by $40 \%$, which really favors nucleation at the edges and reduces locally $\Delta \mathrm{E}$ (as presented in section 2).

Throughout the WW, each dot is subjected to a perpendicular stray field with sufficient amplitude to switch its magnetization individually. Outside the WW, the pulse field is applied 
above the trenches. In sample A, because of the bending of the multilayered structure and the local reduction of anisotropy, the bit reversal probability, when the write pole is above the trenches, does not vanish. This edge effect increases the BER and consequently reduces the WW.

In sample B, the BER tends to zero throughout the WW. Even if e-beam lithography can induce edge effects, by cutting grains at the dots edges for instance, no direct influence was evidenced in the writing performances. Sample B is constituted by $8 \mathrm{~nm}$ diameter weakly coupled grains (about 150 grains per bit). A few grains reversal at the dot edges does not propagate across the entire bit and does not affect the average bit magnetization (the contribution of a few magnetic grains to the total signal is minuscule).

In sample $A$, the strong inter-grain coupling between $20 \mathrm{~nm}$ diameter grains makes the nucleation at the dot edges very critical, because the nucleated bubble can propagate across the entire dot.

\section{Signal-to-noise ratio analyses.}

In addition to writing performances, patterned media must be competitive in terms of SNR.

One way to evaluate the SNR is to compute the read-back signal correlation coefficients after writing a periodic pseudo random sequence (PRBS). Considering two signals of PRBS, v(x) and $\mathrm{v}(\mathrm{y})$, with a periodicity $\mathrm{P}$ such as $\mathrm{v}(\mathrm{x}+\mathrm{nP})=\mathrm{v}(\mathrm{x})$ ( $\mathrm{n}$ is an integer), the noise being typically any fluctuation from the perfect periodic signal, it is possible to calculate the correlation coefficient $\mathrm{R}$ as [26]:

$$
\mathrm{R}(\mathrm{v}(\mathrm{x}), \mathrm{v}(\mathrm{y}))=\frac{\operatorname{Cov}[\mathrm{v}(\mathrm{x}), \mathrm{v}(\mathrm{y})]}{\sigma[\mathrm{v}(\mathrm{x})] \sigma[\mathrm{v}(\mathrm{y})]}
$$


where $\operatorname{cov}[\mathrm{v}(\mathrm{x}), \mathrm{v}(\mathrm{y})]$ is the covariance and $\sigma[\mathrm{v}(\mathrm{x})]$ and $\sigma[\mathrm{v}(\mathrm{y})]$ the standard deviation of both PRBS $\mathrm{v}(\mathrm{x})$ and $\mathrm{v}(\mathrm{y})$ respectively.

Considering several PRBS, the last formula can be extended as:

$$
R(v(x), v(y))=\frac{\frac{1}{N} \sum_{i, j}^{N}\left(v_{i}(x)-\bar{v}(x)\right)\left(v_{j}(y)-\bar{v}(y)\right)}{\sqrt{\left[\frac{1}{N} \sum_{i}^{N} v_{i}^{2}(x)-\left(\frac{1}{N} \sum_{i}^{N} v_{i}(x)\right)^{2}\right]\left[\frac{1}{N} \sum_{j}^{N} v_{j}^{2}(y)-\left(\frac{1}{N} \sum_{j}^{N} v_{j}(y)\right)^{2}\right]}}
$$

And the SNR simply given by:

$$
\mathrm{SNR}=10 \log \left(\frac{\mathrm{R}}{1-\mathrm{R}}\right)
$$

Figure 5 shows a PRBS of 31 bits written in six parallel tracks (a). The corresponding signals along the center of the tracks measured in sample A are superposed within the same graphic (b). Clearly some bits are not correctly written and that some others present a magnetic multidomain structure. Note that the fact a magnetic domain wall can be pinned inside a dot indicates that the reversal mode for sample $\mathrm{A}$ is based on nucleation and propagation processes. To quantitatively analyze the SNR, the bits errors have been removed from the read-back signals, i.e. the corresponding PRBS were not considered. The same measurements have been performed onto sample B and are shown on figure 6 . The writing in that case was more effective and fewer errors were observed. By using formula (4) and the cross correlation coefficients, the SNR was evaluated to be $11.7 \mathrm{~dB}$ and $14.5 \mathrm{~dB}$ for sample A and B respectively. 
It is generally admitted that the main source of noise is due to jitter noise in patterned media [4]. This position noise is linked to the bits position and consequently to the patterning processes. We have already shown by atomic force microscopy measurements a broader distribution in sizes of the dots for fabricated nano-imprinted samples with respect to those fabricated by e-beam lithography [25]. If we do not take into account material fluctuations, and if we assume dots sizes and spacing fluctuations of the same width, i.e $\frac{\sigma_{S}}{S}=\frac{\sigma_{D}}{D}$, it

comes from formula (1), $\frac{\sigma_{D}}{D} \simeq 0.14$ and 0.125 for sample A and B respectively (deduced from the SNR magnitude). Nano-imprint lithography induces more dots sizes and spacing fluctuations than e-beam lithography, which impacts directly the SNR.

\section{Conclusions}

In conclusion, we have investigated and compared the recording performances of two patterned media. By using a quasi-static contact tester with longitudinal write heads, we have measured the bit error rates and the write windows, as well as the signal-to-noise ratio.

The SNR analyses show that nano-imprint lithography induces slightly broader structural distributions than e-beam lithography. Nano-imprint lithography being well adapted for industrial implementation, the technique has to be improved to reduce the standard deviations of structural distributions below 5\%. Concerning the written-in-errors, we have attributed the larger BER of the multilayered material to the microstructure. Local defects at the dots edges constitute potential nucleation centers. If the magnetic grains are strongly coupled (sample A), nucleation can take place at the edges of the dots, followed by propagation of domain wall across the entire dot. In contrast, in patterned media made of weakly coupled grains (sample B), uncontrolled switching probably vanishes when the write pole is located above the 
trenches. To increase thermal stability, which is the challenge of patterned media, bits should be single grain if possible, or composed of several coupled grains. The usage of multilayered material makes this crystallographic property possible. But the edges effects and particularly the widening of the anisotropy distributions at the edges should be avoided. It can be achieved by improving the sharpness of the pre-patterned dots themselves as well as the directivity of the PVD process.

\section{Acknowledgment}

The authors thank Région Rhône-Alpes for financial support. 


\section{References}

[1] D. A. Thompson and J. S. Best. IBM J. Res. DEVELOP. 44, 311 (2000); R. E. Rottmayer et al., IEEE Trans. Magn. 42, 2417 (2006); S. Li, B. Livshitz, H. N. Bertam, E. E. Fullerton and V. Lomakin. J. Appl. Phys. 105, 07B909 (2009).

[2] Stephen Y Chou, Mark S. Wei, Peter R. Krauss, and Paul B. Fischer, J. Appl. Phys. 76, 6673 (1994).

[3] R. L. White, R. M. H. New, R. F. W. Pease. IEEE Trans. Magn. 33, 990 (1997).

[4] M. Albrecht, C. T. Rettner, A. Moser, M. E. Best and B. D. Terris. Appl. Phys. Lett. 81, 2875 (2002).

[5] P. W. Nutter, D. McA. McKirdy, B. K. Middleton, D. T. Wilton and H. A. Shute. IEEE Trans. Magn. 40, 3551 (2004).

[6] J. Lohau, A. Moser, C. T. Rettner, M. E. Best and B. D. Terris. IEEE Trans. Magn. 37, $1652(2001)$.

[7] H.J. Richter, J. Magn. Magn. Matt. 321, 467 (2009).

[8] M. Albrecht, A. Moser, C.T. Rettner, A. Anders, T. Thompson and B.D. Terris, Appl. Phys. Lett. 80, 3409 (2002)

[9] B. Livshitz, A. Inomata, H. N. Bertram and V. Lomakin, J. Appl. Phys. 105, 07C111 (2009).

[10] M.M. Aziz, C.D. Wright, B.K. Middleton, H. Du and P. Nutter, IEEE Trans. Magn. 38, 1964 (2005).

[11] B.D. Terris and T. Thomson, J. Phys. D: Appl. Phys. 38, R199 (2005)

[12] H.J. Richter, A.Y. Dobin, R.T. Lynch, D. Weller, R.M. Brockie, O. Heinonen, K.Z. Gao, J. Xue, R.J.M van de Veerdonk, P. Asselin and M.F. Erden, Appl. Phys. Lett. 88, 222512 (2006). 
[13] M. Asbahi, J. Moritz, B. Dieny, C. Gourgon, C. Perret and R.J.M. van de Veerdonk, to be published.

[14] J. Moritz, B. Dieny, J. P. Nozières, S. Landis, A. Lebib and Y. Chen, J. Appl. Phys. 91, $7314(2002)$

[15] S. Landis, B. Rodmacq and B. Dieny, Phys. Rev. B 62, 12271 (2000).

[16] S. Landis, Ph. D. Thesis "Réseaux de plots magnétiques sub-microniques réalisés à partir de substrats pré-gravés”, Université Joseph Fourier de Grenoble (2001).

[17] J. Moritz, B. Dieny, J. P. Nozières, Y. Pennec, J. Camarero and S. pizzini, Phys. Rev. B 71, 100402(R) (2005)

[18] J.-P. Jamet, S. Lemerle, P. Meyer, J. Ferré, B. Bartenlian, N. Bardou, C. Chappert, P. Veillet, F. Rousseaux, D. Decanini, and H. Launois, Phys. Rev. B 57, 57 (1998).

[19] B.D. Terris, J. Magn. Magn. Matt. 321, 512 (2009)

[20] E. C. Stoner and E. P. Wohlfarth, Philos. Trans. R. Soc. London, Ser. A 240, 599 (1948).

[21] J. Vogel, J. Moritz and O. Fruchart, C. R. Phys. 7, 977 (2006).

[22] M. P. Sharrock, J. Appl. Phys. 76, 6413 (1994).

[23] S. N. Piramanayagam, J. Appl. Phys. 102, 011301 (2007).

[24] T. Thomson, G. Hu, and B. D. Terris, Phys. Rev. Lett. 96, 257204 (2006).

[25] J. Moritz, S. Landis, J-C. Toussaint, P. Bayle-Guillemaud, B. Rodmacq, G. Casali, A. Lebib, Y. Chen, J-P. Nozieres, and B. Dieny, IEEE. Trans. Magn. 38, 1731 (2002).

[26] E. Svedberg, S. Khizroev, and D. Litvinov, J. Appl. Phys. 91, 5365 (2002). 


\section{Table caption}

Table 1:

Magnetic and thickness ( $\mathrm{t}$ ) properties of the investigated samples. The effective anisotropy $\mathrm{K}_{\text {eff }}$ of sample $\mathrm{B}$ is almost one order of magnitude larger than sample $\mathrm{A}$, which influences directly the stability criterion given by the barrier height $\Delta \mathrm{E}$ (in $\mathrm{k}_{\mathrm{B}} \mathrm{T}$ units at room temperature).

\begin{tabular}{|c|c|c|c|c|c|c|}
\hline Sample & Material & $\mathrm{K}_{\text {eff }}\left(\mathrm{J} / \mathrm{m}^{3}\right)$ & $\mathrm{t}(\mathrm{nm})$ & $\Delta \mathrm{E}\left(\mathrm{k}_{\mathrm{B}} \mathrm{T}\right)$ & $<\mathrm{H}_{\mathrm{C}}>(\mathrm{T})$ & $\mathrm{SFD}(\mathrm{T})$ \\
\hline $\mathrm{A}$ & $\mathrm{Co} / \mathrm{Pt}$ & $3 \times 10^{5}$ & 9.6 & 75 & 0.281 & 0.216 \\
\hline $\mathrm{B}$ & $\mathrm{CoCrPt}$ & $9 \times 10^{5}$ & 20 & 200 & 0.694 & 0.318 \\
\hline
\end{tabular}

Table 1. 


\section{Figure Captions}

Figure 1:

a) $2.5 \times 2.5 \mu \mathrm{m}^{2}$ magnetic force microscopy image of sample B. The mono-polar contrasts are associated with a perpendicular-to-plane magnetization. Dots are 100nm diameter, spaced by $200 \mathrm{~nm}$ and are arranged hexagonally.

b) Decreasing field branches of the hysteresis loops of sample A and B measured by magneto-optical polar Kerr effect. The field is applied perpendicularly to the film plane.

Figure 2:

a) GMR signal density map after writing alternating up and down bits in sample B. The dark and light contrasts are associated with the magnetization direction.

b) Corresponding GMR signal centered on a single track in the down-track direction.

Figure 3:

The same bit sequence used in figure 2 has been written several times in sample A. Between each measurement a write current phase shift (y) is added in the down-track direction. The write current is not switched at the same location, and written-in-errors occur for some phase shift. The bit error rate is low for tracks $1,3,5$ and large for tracks 2, 4, 6. In track 3, all bits are well synchronized with the write current. In track 4, where the current phase shift is of about $80 \mathrm{~nm}$ (dots spacing) with respect to track 3, the bits are out-of-phase for most of them. 
Figure 4:

Percentage of synchronized bits $v s$. the phase shift applied to the write pulse in the down track direction for sample A (a) and sample B (b). Data have been extracted from the experiments described in figure 3. The write windows are shown in the figures for a $\mathrm{BER}<5 \%$.

Figure 5:

Pseudo random bits sequences have been written in sample A and scanned with the read-head. The GMR signal density map is given in a) for six parallel tracks. In b) the GMR signal centered on the tracks is plotted. There are some written-in errors and multidomain dots, which have been removed for the signal-to-noise ratio analyses.

Figure 6:

31 Pseudo random bits sequences have been written in sample B. The GMR signal for some tracks is shown in the figure. The signal can be compared as the one presented in figure 5 for sample A. 


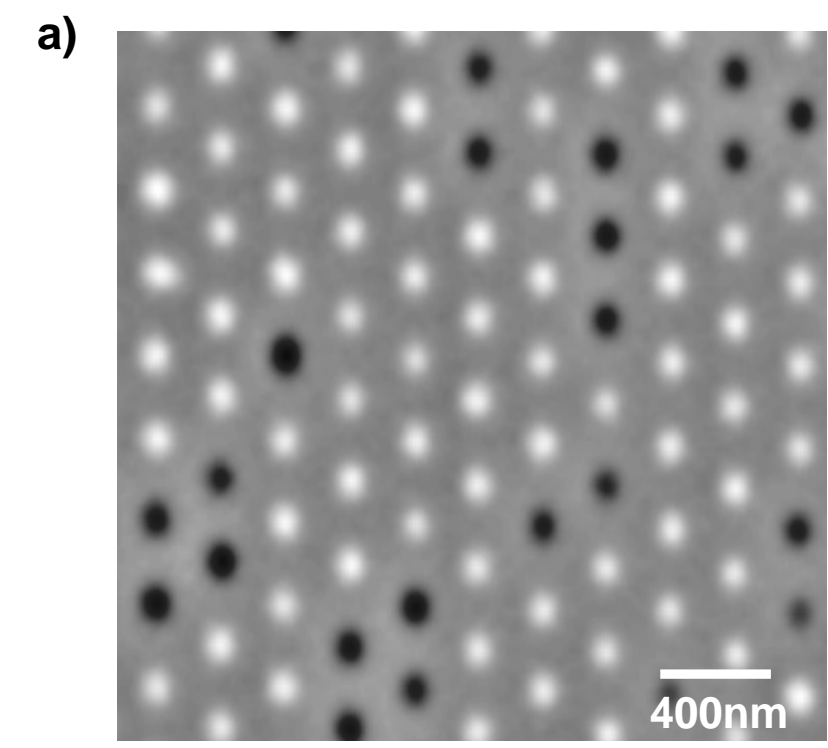

b)

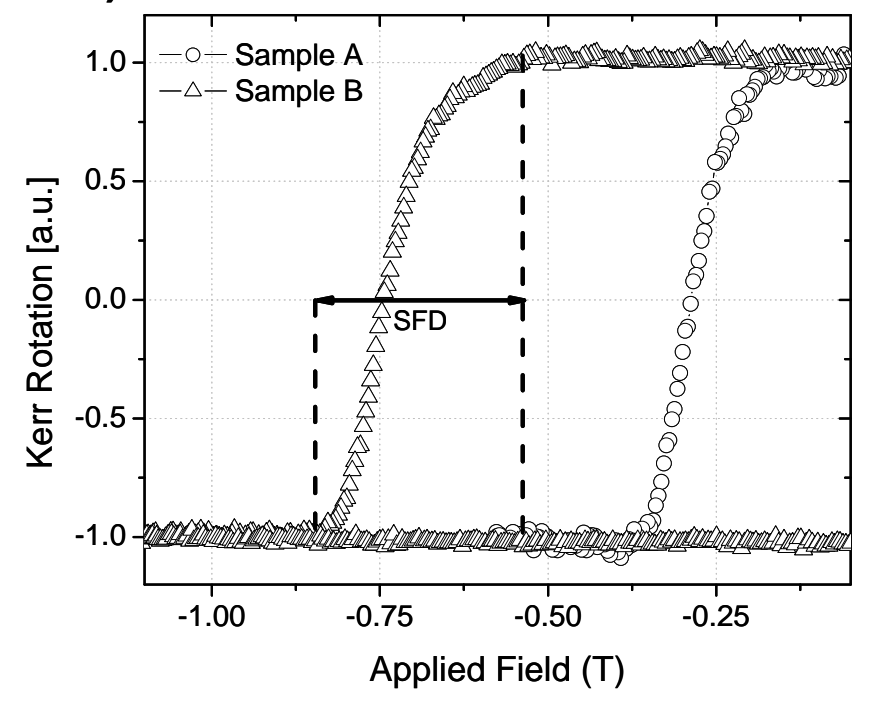

Figure 1 

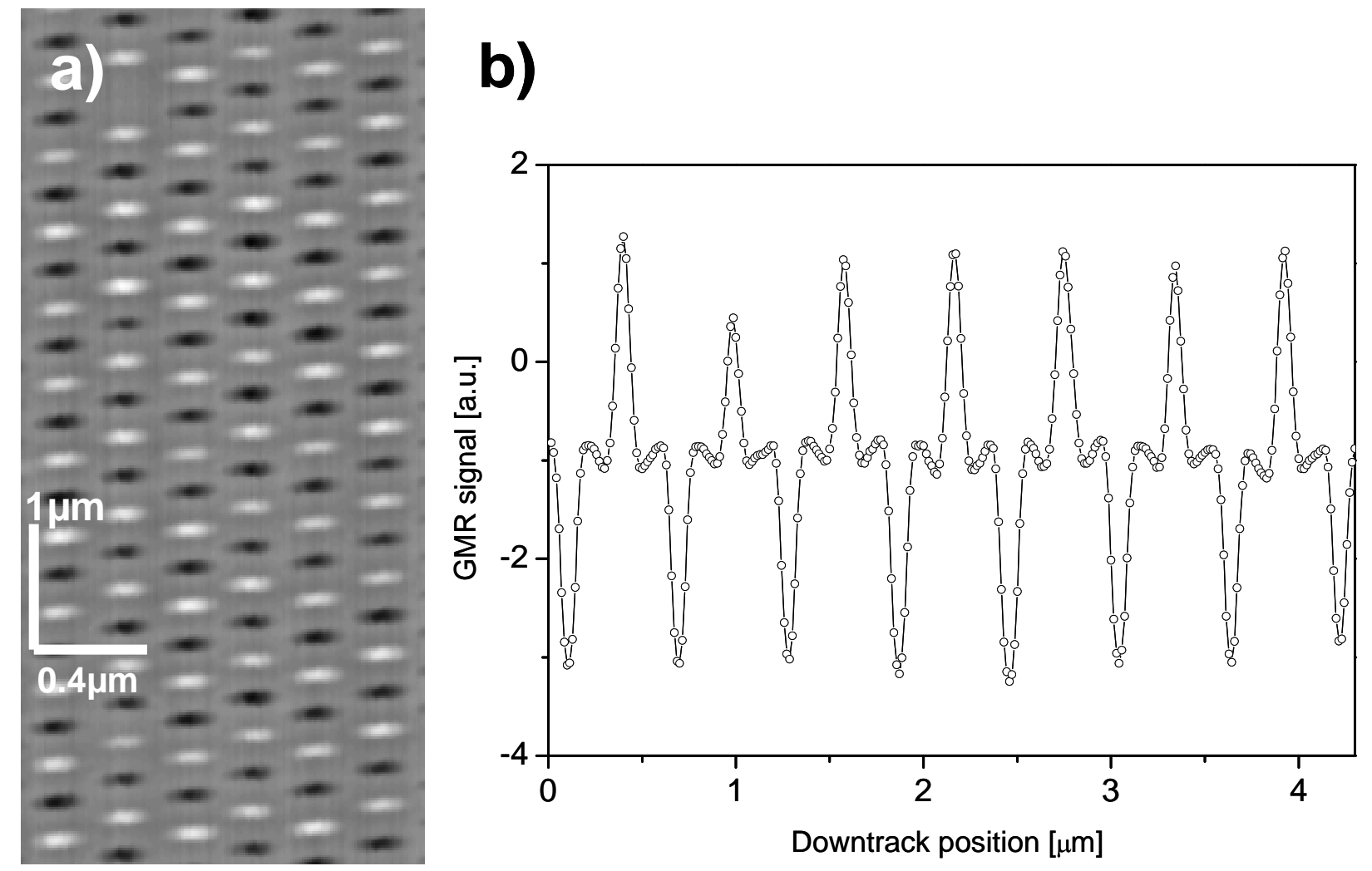

Figure 2 


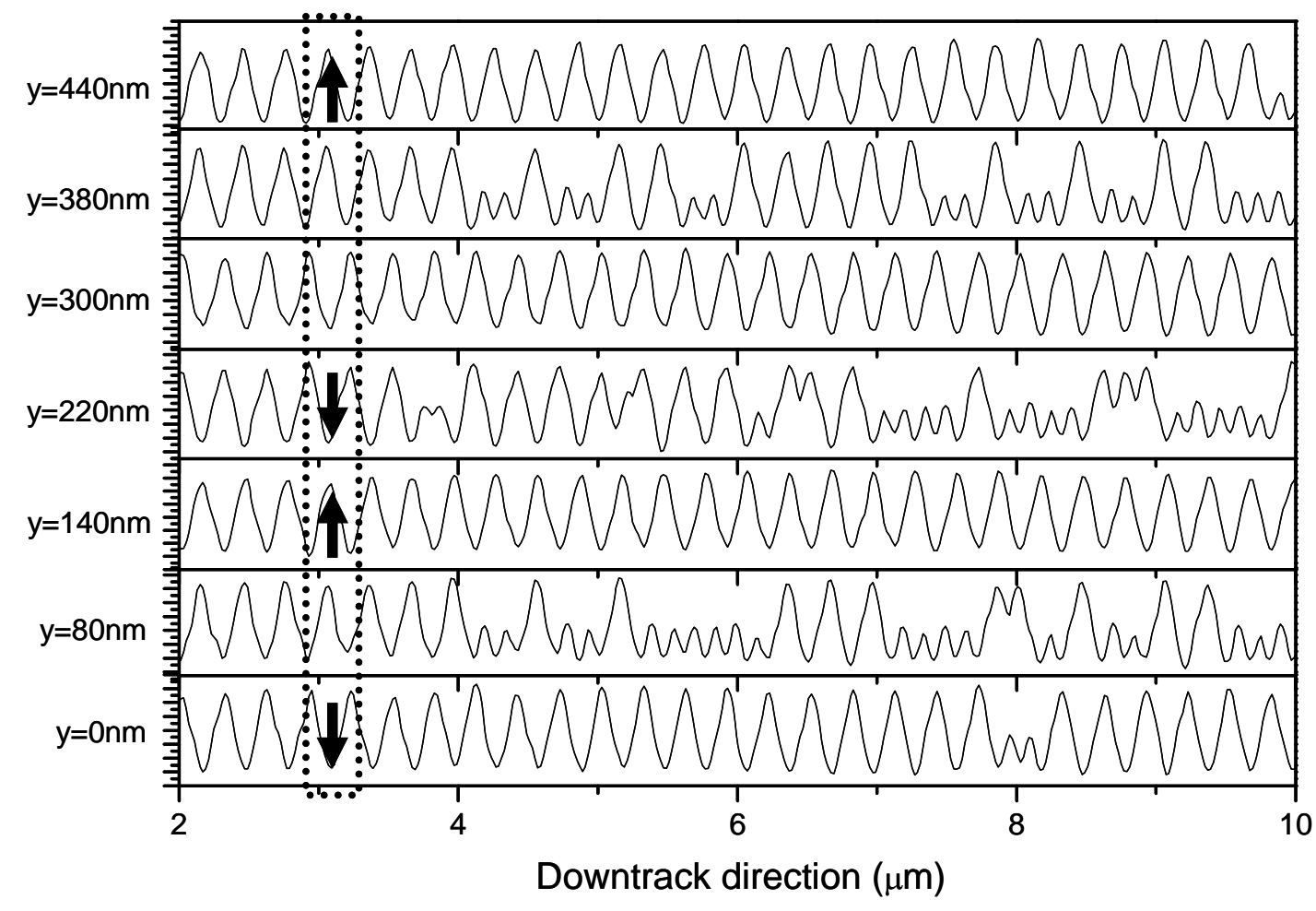

(7)

(6)

(5)

(4)

(3)

(1)

Figure 3 
a)

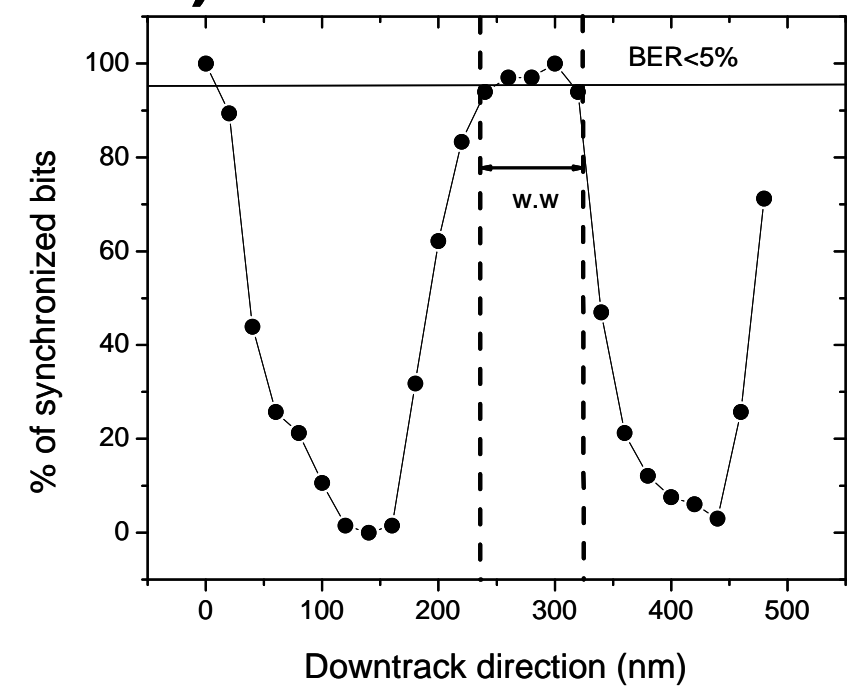

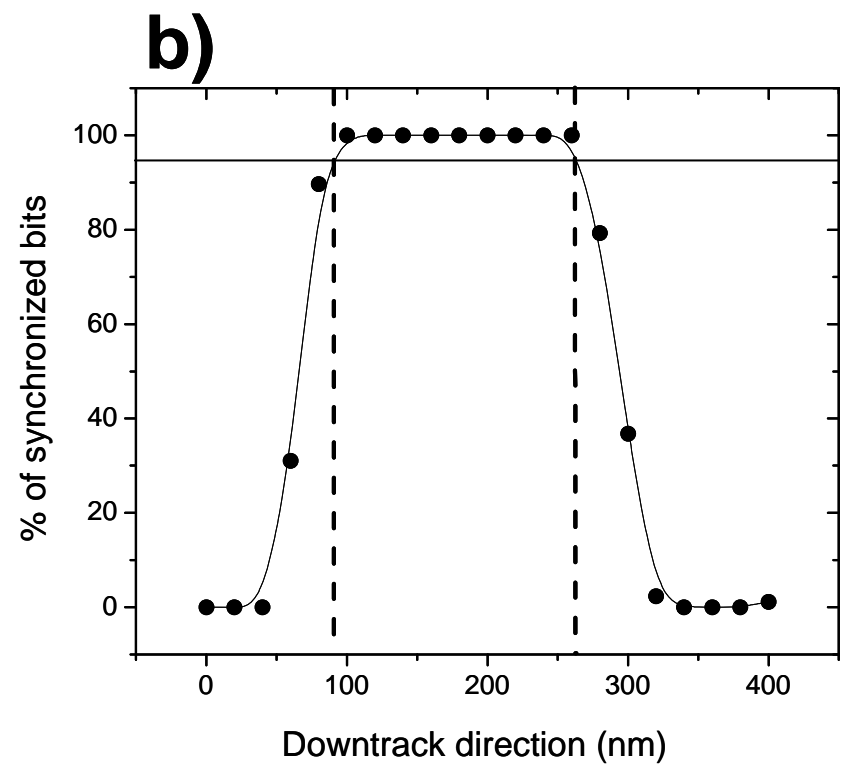

Figure 4 

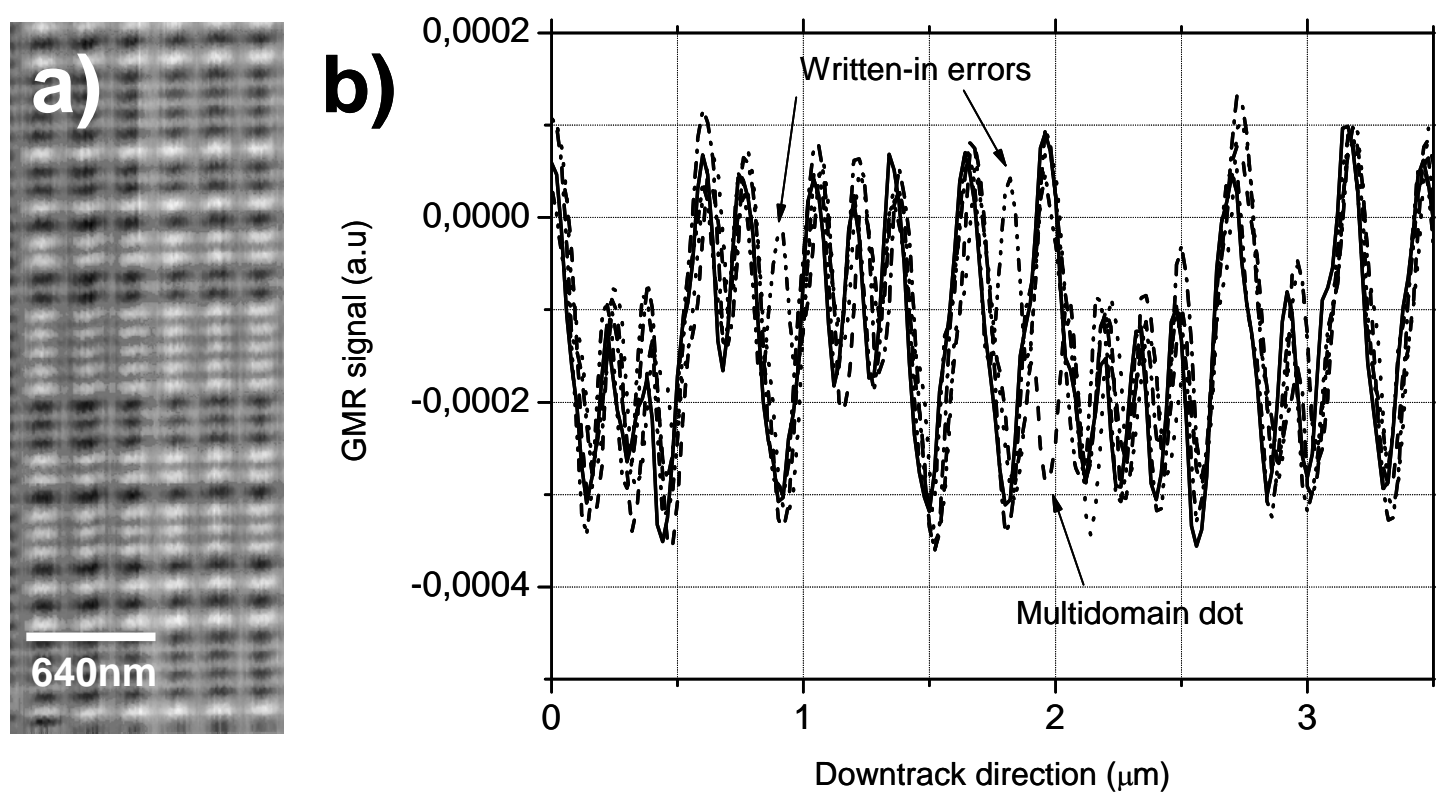

Figure 5 


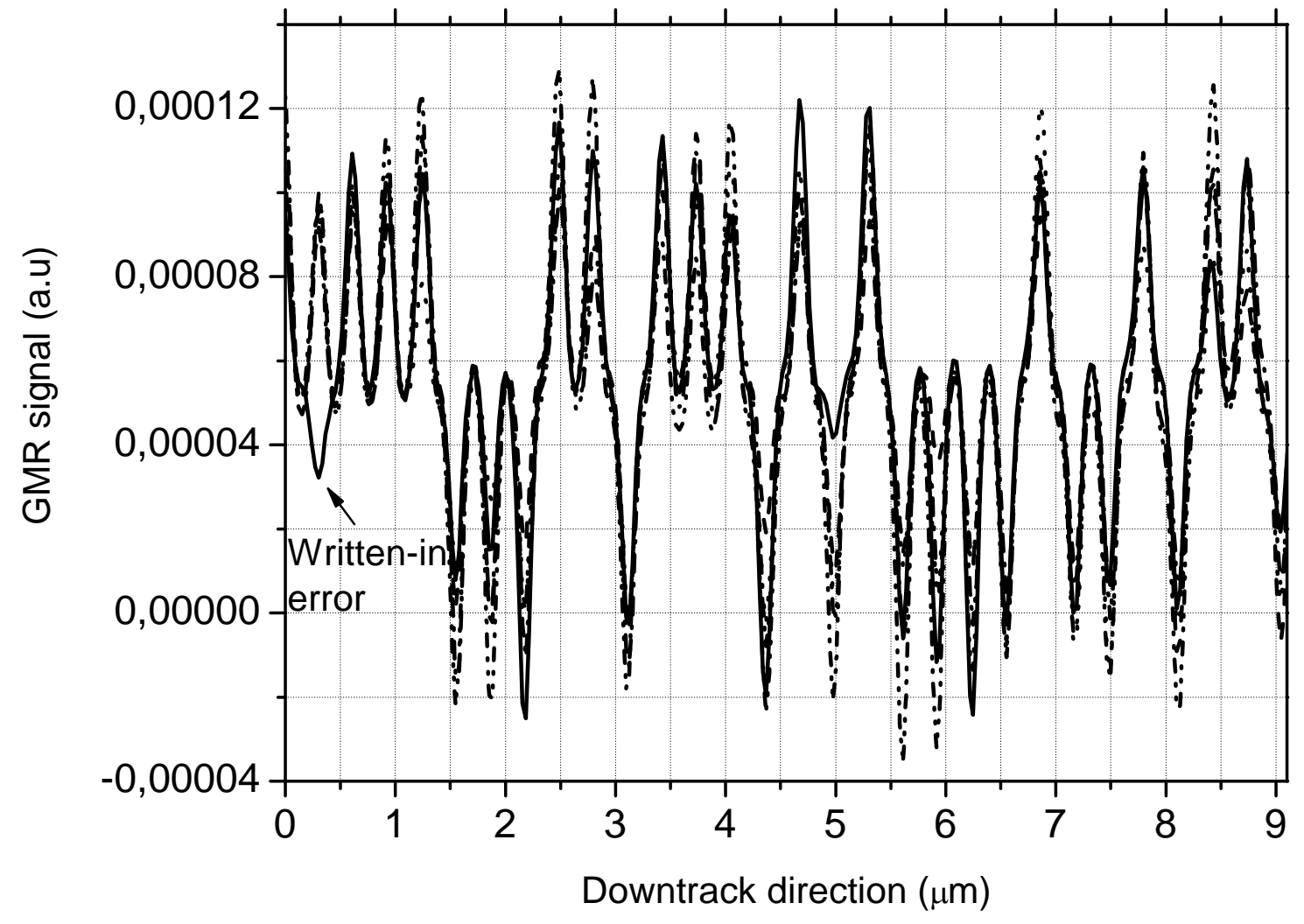

Figure 6 